\title{
Capacity Impacts and Optimal Geometry of Automated Cars' Surface Parking Facilities
}

\author{
You Kong, ${ }^{1}$ Scott Le Vine $\mathbb{D}^{1,2,3}$ and Xiaobo Liu $\mathbb{D}^{1}$ \\ ${ }^{1}$ School of Transportation and Logistics, Southwest Jiaotong University, Chengdu, China \\ ${ }^{2}$ Department of Geography, State University of New York (SUNY) at New Paltz, New Paltz, NY, USA \\ ${ }^{3}$ Imperial College London, Department of Civil and Environmental Engineering, South Kensington SW7 2AZ, UK \\ Correspondence should be addressed to Xiaobo Liu; xiaobo.liu@swjtu.cn
}

Received 3 January 2018; Revised 14 February 2018; Accepted 11 March 2018; Published 26 April 2018

Academic Editor: Zhi-Chun Li

Copyright (C) 2018 You Kong et al. This is an open access article distributed under the Creative Commons Attribution License, which permits unrestricted use, distribution, and reproduction in any medium, provided the original work is properly cited.

\begin{abstract}
The impact of Automated Vehicles (AVs) on urban geography has been widely speculated, though there is little quantitative evidence in the literature to establish the magnitude of such effects. To quantify the impact of the greater precision of automated driving on the spatial efficiency of off-street parking facilities, we develop a mixed integer nonlinear model (solved via a branch-and-cut approach) and present comparisons against industry-standard requirements for human-driving operation. We demonstrate that gains on the order of $40-50 \%$ in spatial efficiency (parking spaces per unit area) are in principle achievable while ensuring that each parked vehicle is independently accessible. We further show that the large majority of these efficiency gains can be obtained under current automotive engineering practice in which only the front two wheels pivot. There is a need for standardized methods that take the parking supply of a city as an input and calculate both the aggregate (citywide) efficiency impacts of automated driving and the spatial distribution of the effects. This study is intended as an initial step towards this objective.
\end{abstract}

\section{Introduction}

Automation technologies for automobiles and other road vehicles are becoming increasingly available commercially, with further advances anticipated in coming years in both fundamental technology and its manifestation as consumer products. Impacts are expected across multiple aspects of the transportation system, including road-network capacity, land use, the disutility of travel time, safety, vehicle-pedestrian interactions, the private-car-ownership business model, public transportation systems, and parking (Fagnant and Kockelman [1], Milanes et al. [2], Malokin et al. [3], Smith et al. [4], Millard-Ball [5], NACTO [6], Lam et al. [7], Wadud et al. [8], and Gucwa, [9]).

AVs' impacts on the parking system are expected to evolve over time. In the long-term, AVs may lead to new forms of vehicle storage, such as zero-occupant circulation to remote parking facilities or continuous on-street zero-occupant circulation while the vehicle's occupant performs his/her activity. Both such concepts would require a large increase in the number of pick-up/drop-off locations, whereas in the prevailing human-driven paradigm a vehicle's parking space is typically also used for pick-up/drop-off activity. In a nearerterm application of automation technologies, self-parking systems which maneuver into a parking space at low speeds are increasingly available commercially (Healey and Mays [10]). There is also evidence of property developers considering the possible obsolescence of parking facilities if vehicle automation leads to lower rates of automobile ownership (Vincent [11]).

The parking system is a major consumer of space in automobile-oriented urban regions; parking appears to account for very roughly three-eighths of the land area of central Los Angeles, for instance (see discussion in Manville and Shoup [12]). Recent work by McCahill et al. [13] quantifies rapid increases in parking supply in US cities in the late 20 th/early 21 st centuries. Industry estimates are that the cost of providing parking is in the range of $\$ 5,000-\$ 50,000$ on a per parking space basis (National Parking Association [14]). Thus in the aggregate parking supply and operations have broad consequences for urban geography. 


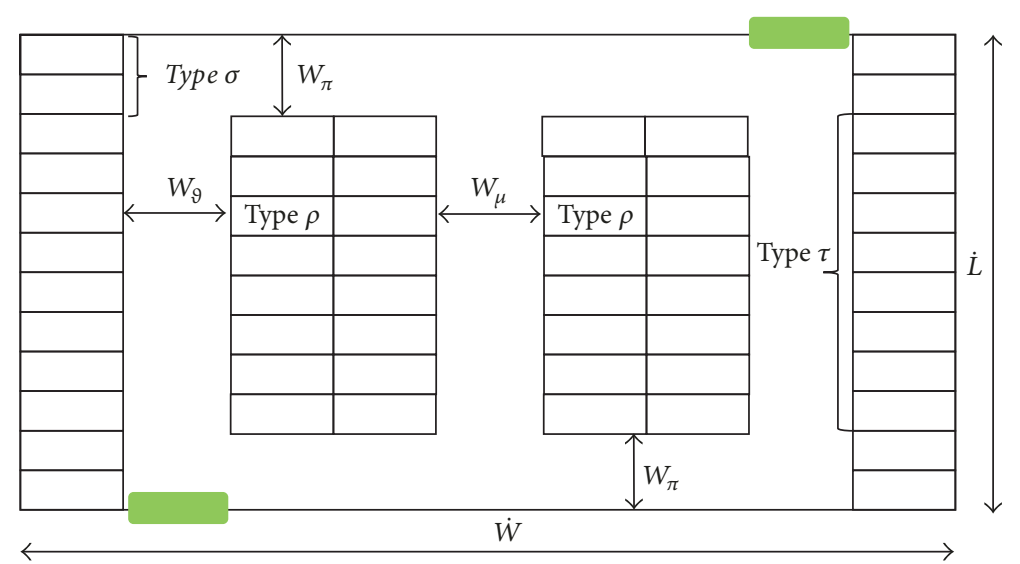

(a)

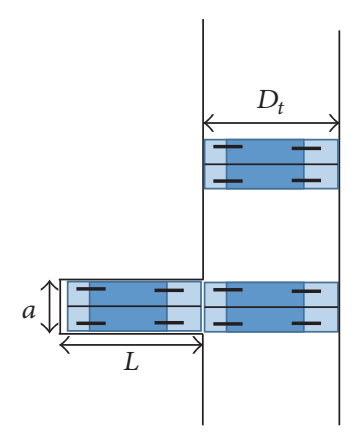

(d)

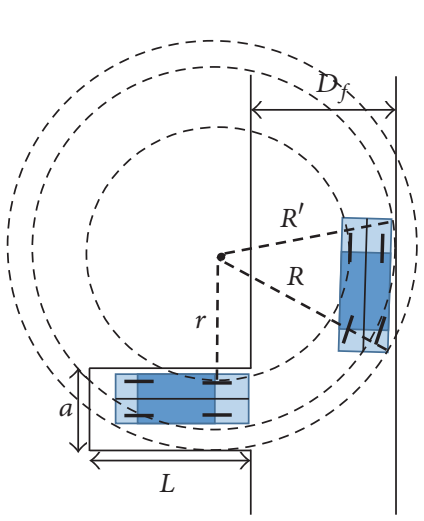

(b)

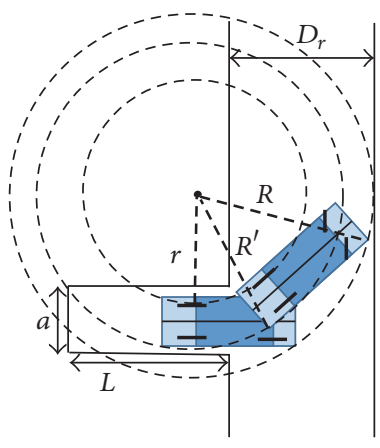

(c)

Figure 1: Panel: (a) General parking layout strategy employed in this study; (b) "front-in" parking maneuver; (c) "reverse-in" parking maneuver; (d) "translation" parking maneuver.

This paper's specific focus is the impacts of AVs on the spatial efficiency (vehicles stored per unit area) of parking facilities (NB: we neglect the efficiency of internal circulation provided that the maneuvers can in principle be performed, in order to focus exclusively on spatial efficiency). This is an important research question, because any increase in the spatial efficiency of vehicle storage would affect the density of land use and hence the valuation of urban land, independent of any change in the location of vehicle storage (see previous paragraph). Our objective in this study is to provide a contribution by directly addressing the narrow research question of how automated cars will affect vehicle storage capacity and thereby provide an incremental advance towards the broader research question "How will automated cars affect urban geography via the mechanism of impacts to the vehicle-parking ecosystem?"

To address this topic, in this study we develop a mixed integer nonlinear programming (MINLP) approach to compare the spatial efficiency of human-driven and AV surface parking facilities and subsequently implement it. We present numerical results considering three types of parking maneuvers ("front-in," "reverse-in," and "translation") and facility sizes from $100 \mathrm{ft} \times 100 \mathrm{ft}(10,000 \mathrm{sq} f \mathrm{ft})$ to $1000 \mathrm{ft} \times$ $1000 \mathrm{ft}(1,000,000 \mathrm{sq} \mathrm{ft})$, including both square and rectangular shapes of the parking facility. We note that there are an infinite number of layout strategies for any given parking facility; the specific layout strategy that we employ in this study is shown in Figure 1(a). We leave the study of alternative layout strategies as an item for future research.

The remainder of this paper is organized as follows: Section 2 discusses earlier literature on automation technology in parking facilities. Section 3 introduces the proposed mixed integer nonlinear programming optimization framework for modeling the spatial efficiency of human and automated parking, and Section 4 presents results from numerical analyses. Section 5 concludes the paper with a brief summary and discussion of future research needs.

\section{Background}

The prevailing automobile-parking paradigm in large, formal facilities involves a human driver circulating within the parking facility and ultimately maneuvering their vehicle into one of the facility's demarcated parking spaces. The human driver typically gathers information regarding the availability of parking spaces via line-of-sight and performs the parking maneuver manually. Alternative concepts include "smart vehicular technology" such as self-parking systems in which the technology is entirely vehicle-borne, "smart 
infrastructure" within the parking facility, to provide beyondline-of-sight (BLOS) information regarding parking space availability, and "parking information sharing," to provide parking variable message signs (parking VMS) (Sun et al. [15], Ni and Sun [16]). One method frequently employed to analyze such information provision is via microscopic parking simulation (Horni et al. [17], Dia [18], and Geng and Cassandras [19]). There are also examples of parking facilities in which the physical maneuvering of the vehicles is performed mechanically by the facility's "smart infrastructure." In general, parking is an area of rapid technological development (Dayan [20], Levine et al. [21], Kuhlman et al. [22], Belzner and Pedron [23], Urbach [24], and Elie and Rhode [25]). Concepts have also been proposed to allow all four of an automobile's wheels to independently pivot, allowing translational motions, such as a parallel parking maneuver in which an automobile moves only laterally (not longitudinally) into the parking space (Nissan [26], Kroger [27], and Schaub et al. [28]). This is in contrast to contemporary automotive design, in which only the front two wheels pivot and the position of the rear wheels remains fixed.

Seminal early work by Ricker [29] established the geometry of automobiles' parking maneuvers and the relationship between parking space width and circulation aisle width. Later work by Chen and Schonfeld [30], Iranpour and Tung [31], and Chodash [32] analyzed the spatial efficiency of different angles of parking with human drivers, with Chen and Schonfeld [30] addressing optimality of parking angle for an arbitrarily large parking facility, Iranpour and Tung [31] considering allocating parking spaces of various sizes to different-sized vehicles, and Chodash [32] focusing on a small number of specific facility dimensions and parking angles. More recently, Blackburn [33] investigated the kinematics of parallel parking and "front-in" parking maneuvers. There is also a rich body of literature on the impacts of parking policies and information provision (Ferguson [34], Shoup [35], Ni et al. [36], Arnott et al., [37], Chester et al. [38], and Chen et al. [39]).

While there is speculation that AVs will impact on parking through a variety of mechanisms, with very few exceptions the literature consists of educated guesswork about the impacts of AVs on parking supply and demand, rather than objective quantitative findings.

A number of recent macroscale travel-forecasting efforts have attempted to quantify the combined effects of AVs on all aspects of the transportation system, including parking impacts. Kim et al. [40] and Childress et al. [41] attempted to do so by assuming that parking costs would decrease and both studies explicitly noted increased spatial efficiency of parking facilities as a mechanism for lowering the costs of parking. Arnott et al. [37] likewise assumed a reduction in vehicle-kilometers of travel due to a reduction in "hunting for parking."

Among studies that have focused in detail on AVs' parking, Schaub et al. [28] address the motion-planning problem for an individual $\mathrm{AV}$ to perform a translational parking maneuver. Three prior studies have studied aspects of the spatial efficiency of AV parking (Ferreira et al. [42], Timpner et al. [43], and Estepa et al. [44]). Ferreira et al. investigated "stacked" storage of AVs, in which a parked vehicle blocked in by other vehicles would be accessed by the blocking vehicles shunting themselves (without human input). Ferreira and colleagues make an assumption that the width of a parking space would be reduced by $20 \%$ as an input to their work, rather than an output of an optimization process as in the present study. Timpner et al. investigated a similar scenario of blocked-in vehicles and automatic shunting, with no treatment of possible impacts on the size of parking spaces. Estepa and colleagues analyze the special case of storing AVs in "double-parking" locations alongside rows of parking spaces whose occupying vehicles would be "blocked in"; the impact ascribed to AVs arises from an assumption that they would be able to communicate and maneuver as necessary without human control to allow a blocked-in parked vehicle to exit. The authors propose a communications protocol to enable the coordinated movements required for the AVs that are in the "blocking" double-parked row. Estepa et al. treat the physical maneuverability of AVs as same as humandriven vehicles and introduce the stimulus of vehicle-tovehicle communications; by contrast in this study we analyze the physical maneuverability of AVs and make no assumption regarding the impact of $\mathrm{V} 2 \mathrm{~V}$ communication. Our approach allows the effect of AVs' enhanced physical maneuverability relative to human drivers to be isolated from other effects due to more-speculative aspects of vehicle automation or connectivity.

The original contribution of this paper is therefore that we present the first systematic framework for quantitatively analyzing the spatial efficiency gains from AVs performing more precise parking maneuvers than humans do within surface parking facilities, respecting the fundamental geometric constraints of vehicle parking. To do this, we evaluate a range of automated driving maneuvers: (1) single-motion "frontin" maneuvers (in which the vehicle front enters the parking space first), (2) single-motion "reverse-in" maneuvers (vice versa), (3) "front-in" maneuvers with multiple "toing/froing" motions, and (4) "translation" maneuvers that require the rear wheels to pivot independently of the front wheels (which standard contemporary automotive design does not accommodate).

\section{Methods}

This study consisted of two distinct research tasks: (1) calculation of the minimum geometric requirements for parking spaces and circulation aisles under various operational concepts and (2) development of a constrained-optimization framework for calculating the number of parking spaces that can be accommodated within a single-level facility of a given length and width. Sections 3.1 and 3.2 describe these tasks in sequence; Section 4.1 then describes this study's numerical analysis. We first, however, introduce the set of assumptions that underpin our analysis:

(1) Throughout this analysis, we work with a single-story parking facility, with no interior obstructions (e.g., no structural columns for overhead buildings, no light poles). We note that for existing parking facilities that 
have rigid internal obstructions (e.g., parking garages with structural columns), the capacity impacts may be quite different. Ricker [29], for instance, noted that capacity in many such facilities experienced capacity losses between one-fourth and one-third as the dimensions of automobiles increased between the 1920 s and 1940s.

(2) We work with surface parking facilities that have perfect square or rectangular dimensions; we do not consider other shapes.

(3) We treat all AVs as having identical dimensions and physical maneuvering capabilities. Vehicle length $\left(19^{\prime}\right)$, width $\left(7^{\prime}\right)$, wheel locations (for the Passenger Car design vehicle in (AASHTO 2011), the wheelbase is $6^{\prime}$ for both the front and rear axles; the front overhang (from front axle to front bumper) is $3^{\prime}$ and the rear overhang is $5^{\prime}$ ), and turning radius $\left(17.9^{\prime}\right)$ are sourced from the "Passenger Car" design vehicle in (AASHTO [45]). We note that these dimensions are somewhat larger than an average-sized automobile (page 2-1 of AASHTO 2011): "A Design Vehicle Has Larger Physical Dimensions and a Larger Minimum Turning Radius Than Most Vehicles in Its Class."

(4) We assume near-zero error in AVs' localization and physical maneuvering, which implies that near-zero clearance is required at points of potential physical conflict between a maneuvering vehicle and the locations on which it cannot encroach. To the extent that positioning errors exist, larger errors would lead to more generous (larger) dimensions of parking spaces and circulation aisles. Therefore, the spatial efficiency values that we report should be interpreted as theoretical maximum values, with achieved values somewhat less spatially efficient. We also note that the Passenger Car design vehicle is larger than most automobiles, meaning that the dimensions that we calculate will allow nontrivial clearances for most cars in the current car population's distribution of vehicle size.

(5) We assume that each vehicle needs to be independently accessible at all times (i.e., no parked vehicle can be "blocked in"). This is in contrast to the stimulus assumed in (Estepa et al. [44]); that study specified the only unique capability of AVs to be that parked vehicles could be blocked in and that a pervasive vehicleto-vehicle communications mechanism would lead to cooperative behavior on the part of one or more AVs to allow the blocked-in vehicle to exit.

(6) We assume that two entrance/exit locations are needed to access the surface parking facility.

(7) We assume that there is not a need for vehicles larger than the Passenger Car design vehicle from AASHTO [45] to circulate within the parking facility (e.g., fire trucks). We note that AASHTO [45] recommends that " $A$ passenger car may be selected [as the design vehicle] when the main traffic generator is a parking lot or series of parking lots" (page 2-2).
(8) We assume that AV occupants enter/exit the vehicles somewhere outside of the parking space, so that the width of parking spaces is not constrained by the need to open car doors.

(9) We specify that all maneuvers are either tangents or are arcs of fixed-radius circles, with the minimum turning radius per AASHTO [45] (see Assumption \#3).

(10) We assume that when turning between circulation aisles oriented north-south and east-west (and vice versa), AVs are permitted to temporarily encroach onto the lane for traffic moving in the opposite direction.

(11) We assume that all parking spaces in a standard double-loaded row are of the same dimension.

(12) We study surface parking facilities of $100^{\prime} \times 100^{\prime}$ dimensions and larger. This is based on a calculation of $85.5^{\prime} \times 85.5^{\prime}$ as the minimum square facility size to include two rows of parking with "human driver sized" dimensions. The analysis of facilities smaller than this, at which idiosyncrasies of entry/exit locations become dominant, is left as an item for future research.

(13) We specify that the east-west circulation aisles along the northern and southern facility boundaries must accommodate two-way traffic; for human-driver layouts this implies a minimum width of $24^{\prime}$ (per ULI [46]) and for AV layouts this implies a width of $14^{\prime}$ (twice the vehicle width). We do not impose this restriction on north-south circulation aisles; in practice this means that in AV layouts where north-south circulation aisle widths of less than $14^{\prime}$ are required, the consequence is that only one-way circulation could be permitted in the north-south aisles.

3.1. Minimum Geometric Requirements. We evaluate three types of parking maneuvers by AVs: "front-in" motions, "reverse-in" motions, and "translation" (see Figure 1).

For "front-in" and "reverse-in" maneuvers, the geometry depends on the number of forward and reverse "toing-andfroing" motions, with the lower limit being one single motion to enter the parking space and no natural upper limit. In order to put lower and upper boundaries on the spatial requirements, our strategy is therefore to use the "singlemotion" geometry shown in Figure 1 to provide an upper limit on the spatial requirements and an infinite number of toing/froing motions as the lower limit. In the latter case, for "front-in" parking the absolute minimum width of the circulation aisle is simply the diagonal dimension of the vehicle if it is assumed that the $\mathrm{AV}$ must circulate in the forward direction and then can perform an infinite number of toing-and-froing motions to enter the parking space.

For the translation parking maneuver, we specify that the vehicle performs any needed rotational movements prior to entering the parking facility, and hence the circulation aisles need only be as wide as the vehicle length. Therefore, while parking facilities that are designed on an assumption that all 
vehicles will perform translation parking maneuvers would require changes to automotive engineering, the "translation" parking maneuver provides an upper boundary on a rectangular parking facility's achievable spatial efficiency (subject to the assumptions noted earlier in this section).

The "translation" maneuver to enter a parking space cannot be readily performed by mass-market automobiles today, because it requires that all four wheels are capable of rotating full 90 degrees. Such vehicle-design is problematic for human drivers, who are trained to operate vehicles whose front wheels pivot and rear wheels do not. It may become more feasible within the automated driving domain, as the complex wheel-maneuvering logic would be algorithmically controlled. However, we note that if we relax the assumption that a car whose rear wheels do not pivot must travel forward in a circulation aisle, the possibility is raised of the vehicle orienting itself in a circulation aisle perpendicular to the aisle if the aisle is an arbitrarily small amount larger than the vehicle's length. The vehicle can then in principle perform an arbitrarily large number of toing-froing motions to allow it to move incrementally down the circulation aisle towards its target parking space and to then enter the parking space either front-in or reverse-in without performing a turning maneuver. Thus in the limit a fixed-rear-wheels car could navigate to and enter a parking space designed for "translation" motion but would require it to navigate the circulation aisle via performing an arbitrarily large number of toing/froing motions rather than simple driving forward down the aisle. We note this possibility, but for the remainder of this paper we neglect this strategy for circulating within the parking facility, which would be highly inefficient in terms of traffic circulation.

We sourced the standard dimensions of parking spaces designed for use by human drivers from the relevant literature. ULI [46] provides standard parking space dimensions of $8.67^{\prime}$ width and $19.75^{\prime}$ length for low-turnover spaces, with a corresponding aisle-width dimension of $25^{\prime}$. ULI [46] indicates a width of $9^{\prime}$ for high-turnover spaces, with the same space-length and aisle-width dimensions. In the remainder of this paper, for convenience we employ the terms "human small" and "human large" to refer to these low-turnover and high-turnover dimensions, respectively.

The notation employed throughout this paper is summarized in Notations.

Ricker [29] documented relationships for the minimum spatial requirements for front-in and reverse-in parking maneuvers. The relationships derived by Ricker are adapted as in (1) and (2), respectively, subject to the following specifications: clearances are set to zero, parking angle is set to 90 degrees, and the design vehicle is defined (consistent with AASHTO [45]) to be a perfect rectangle (rather than to have rounded corners as is originally specified in Ricker's work).

$$
\begin{aligned}
& D_{f}=R^{\prime}+\sqrt{R^{2}-\left(r+t+O_{s}+\left(a_{i}-w\right)\right)^{2}} . \\
& D_{r}=R-\sqrt{\left(r-O_{s}\right)^{2}-\left(r-O_{s}-\left(a_{i}-w\right)\right)^{2}}
\end{aligned}
$$

$D_{f}$ and $D_{r}$ denote the minimum circulation aisle width for front-in and reverse-in maneuvers, respectively. For both front-in and reverse-in parking maneuvers, it can be seen that there is a dependency between aisle width $D$ and space width $W$. In subsequent analysis (see Section 3.2), we optimize this trade-off (i.e., maximize the number of parking spaces) for each AV-driving layout that we analyze.

Employing (1) and (2), it can be determined that the minimum aisle width is $32.6^{\prime}$, for "front-in" maneuvers using the human-driven "small" parking space width and 31.9" using the human-driven "large" parking space width. For "reverse-in" maneuvers, the relevant calculations for "small" and "large" human-driven parking space widths are $18.7^{\prime}$ and $18.1^{\prime}$, respectively. It can be seen that both values for humandriven "front-in" maneuvers are comfortably larger than the standard aisle width $\left(25^{\prime}\right.$ for two-way traffic and doubleloaded 90-degree parking rows) for human-driven parking facilities, meaning that contemporary parking facilities are not designed for drivers of larger-than-average automobiles to enter into the parking spaces in a single "front-in" motion without encroaching onto other parking spaces.

3.2. Mixed Integer Nonlinear Optimization. The MINLP model to maximize the number of parking spaces is based on the facility-layout strategy shown in Figure 1(b). The objective function is the sum of three sets of parking spaces:

$$
\max C=n_{\rho}+n_{\sigma}+n_{\tau} .
$$

$n_{\rho}$ denotes the number of parking spaces in the interior rows, and $n_{\sigma}$ denotes the number of parking spaces in the exterior rows that are aligned with the east-west circulation aisles along the northern and southern borders of the facility. $n_{\tau}$ denotes the number of spaces in the exterior rows that are not along the surface facility's northern or southern boundaries. The distinction between the $\sigma$ and $\tau$ types of parking spaces is that the spaces in the $\sigma$ type always have a width of $7^{\prime}$ (i.e., the vehicle width) regardless of the width of the northsouth circulation aisles, whereas the width of the $\tau$ type spaces is subject to the minimum-width constraint (in the front-in and reverse-in scenarios) imposed by the required turning maneuver to enter the space, in which the minimum width of the parking space interacts with the width of the aisle.

The objective function in (3) is subject to the following constraints:

$$
\begin{aligned}
& \frac{a_{\rho} n_{\rho}}{2 * X_{\rho}+X_{\rho, s}} \leq \dot{L}-2 * W_{\pi} \\
& a_{\sigma} n_{\sigma}+a_{\tau} n_{\tau} \leq 2 * L \\
& 7 \leq a_{i} \leq 21 \quad \forall i=(\rho, \sigma, \tau) \\
& 2 L X_{\epsilon, s}+2 W_{\vartheta}+2 L X_{\rho}+W_{\rho}\left(X_{\rho}-1\right)+L X_{\rho, s} \\
& \quad+W_{\rho} X_{\rho, s} \leq \dot{W} \\
& 0 \leq X_{\rho, s} \leq 1 \\
& X_{\epsilon, s}=2
\end{aligned}
$$




$$
\begin{aligned}
& n_{\sigma}=8 \\
& W_{\vartheta}=\max \left(Y_{\epsilon}, W_{\mu}\right) .
\end{aligned}
$$

Constraint (4) accounts for the fact that the double-loaded rows cannot contain more parking spaces than can fit within the total north-south dimension of the facility minus the space needed for the two-way east-west circulation aisles at the northern and southern facility boundaries.

Constraint (5) specifies that the number of spaces of types $\rho$ and $\tau$ are constrained by the length of the parking facility.

Constraint (6) governs the range of parking space widths that are searched in the optimization. The minimum width $\left(7^{\prime}\right)$ is the vehicle width; the maximum width is the critical space width after which further increases have no effect on the corresponding aisle width. Thus there is an interaction between space width and aisle width only within the range shown in Constraint (6).

Constraint (7) ensures that the facility width is at least as large as the space required by the exterior parking rows, the interior parking rows, and all necessary north-south circulation aisles.

Constraint (8) ensures that there can be only one singleloaded interior row; if there were sufficient space for a second interior row there would be one double-loaded interior row rather than two single-loaded interior rows.

Constraint (9) specifies that there are exactly two singleloaded exterior rows: one each along the eastern and western boundaries of the facility.

Constraint (10) specifies that there are exactly eight parking spaces of type $\sigma$. Two such spaces are located at each of the four corners of the facility; these are the spaces in the exterior rows that are aligned with the east-west circulation aisles. Finally, Constraint (11) ensures that for circulation aisles that have interior parking on one side (spaces of type $\rho$ ) facing exterior parking on the aisle's other side (spaces of type $\tau$ ), the aisle's width is the maximum of the aisle width required to access both of these types of spaces.

3.3. Solution of MINLP. We employed the LINDOGLOBAL solver within Lingo software [47] to solve the MINLP problem described in the previous section. This optimization algorithm is designed to identify optima for both convex and nonconvex MINLPs, using a branch-and-cut approach [48] based on Linear Planning for bounding. Branching is performed for subproblems with feasible solutions where nonconvex constraints are present or the LP relaxation has a fractional solution [49]. The results produced using the LINDOGLOBAL optimizer were verified via an exhaustive search method (using Matlab software) with the search space discretized with increments of 0.1 feet for all parking space and aisle dimensions between their calculated theoretical lower and upper bounds.

\section{Numerical Analysis}

In this section we first (Section 4.1) introduce the experimental design of the numerical analyses, which we solve by using Lingo software [47] and then in Section 4.2 present the results.

4.1. Parameters of Numerical Analysis. The numerical analyses encompass six different operational requirements, two for human drivers (the "small" and "large" dimensions introduced in Section 3.1) and four for AVs (AV front-in, AV reverse-in, AV toing/froing, and AV translation).

For each of these operational requirements we calculate the maximum number of parking spaces for squares with sides of $100^{\prime}$ up to sides of $1000^{\prime}$, in $1^{\prime}$ increments (and no internal obstructions).

We then quantify the effect of asymmetry in facility dimensions by analyzing rectangular facilities having the same area as squares of $250^{\prime} \times 250^{\prime}(62,500 \mathrm{sqft}), 500^{\prime} \times$ $500^{\prime}(250,000 \mathrm{sq} f \mathrm{ft})$, and $1000^{\prime} \times 1000^{\prime}(1,000,000 \mathrm{sq} \mathrm{ft})$. For rectangular facility shapes, we consider all rectangles between $\dot{L}=100^{\prime}$ (with the corresponding value of $\dot{W}$ to yield the desired facility area) and $\dot{W}=100^{\prime}$ (with the corresponding value of $\dot{L}$ to yield the desired facility area).

Results from these scenarios, and comparisons between them, are found in the next section.

4.2. Analysis of Results. In advance of the numerical results presented later in this section, we first begin with illustration of the optimal layouts for a representative square facility with $500^{\prime}$ sides. Figures 2(a) and 2(b) show the optimal layouts using the "small" (a.k.a. "low-turnover," at (a)) and "large" (a.k.a. "high-turnover," at (b)) standard dimensions for human-driving. (c), (d), (e), and (f) show layouts for the four AV parking maneuvers ("front-in," "reverse-in," "toing/ froing," and "translation"). The number of parking rows is identical; the efficiency of the "small" relative to the "large" space dimensions (790 spaces versus 760 spaces) is due to the larger number of spaces per row (e.g., 52 versus 50, in the interior rows).

The AV "front-in" facility (Figure 2(c)) has wider aisles than the human "small" facility and hence one little doubleloaded interior row; however the vehicle storage capacity is larger (814 versus 760) due to a larger number of spaces per row. The AV "reverse-in" facility has 1036 spaces, with both an additional single-loaded row relative to the human "small" layout and a larger number of spaces per row (e.g., 64 versus 52, in the interior rows). The AV "toing/froing" and "translation" layouts have the same number of rows as each other and also the AV "reverse-in" layout; however both the "toing/froing" and "translation" layouts have 67 spaces per interior row (compared to 64 for "reverse-in"). These two layouts have the same overall number of parking spaces; it can be seen that the difference between them is that the rightmost aisle is wider for the "translation" layout (but not wide enough for an additional single-loaded row), because all aisles to the left are marginally narrower.

Figure 3 shows the average spatial requirement of each parking space, for squares with sides $100^{\prime}$ to $1000^{\prime}$ and in $1^{\prime}$ increments. Spatial requirement is defined as the overall facility area divided by the parking capacity of the facility. The two curves in (a) depict the spatial requirements for parking 


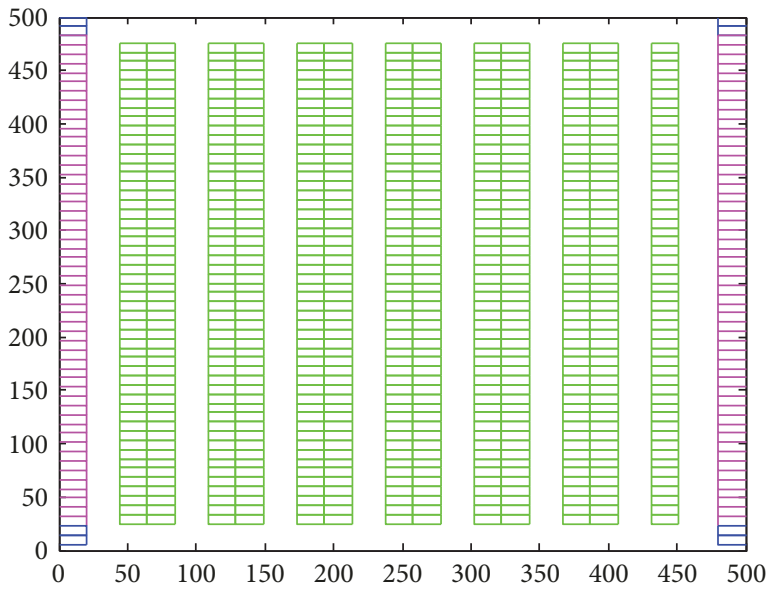

(a)

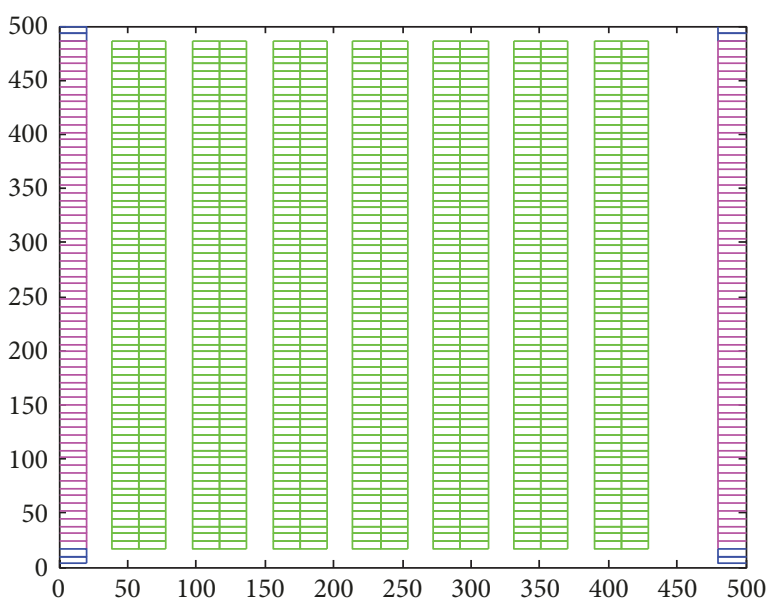

(f)

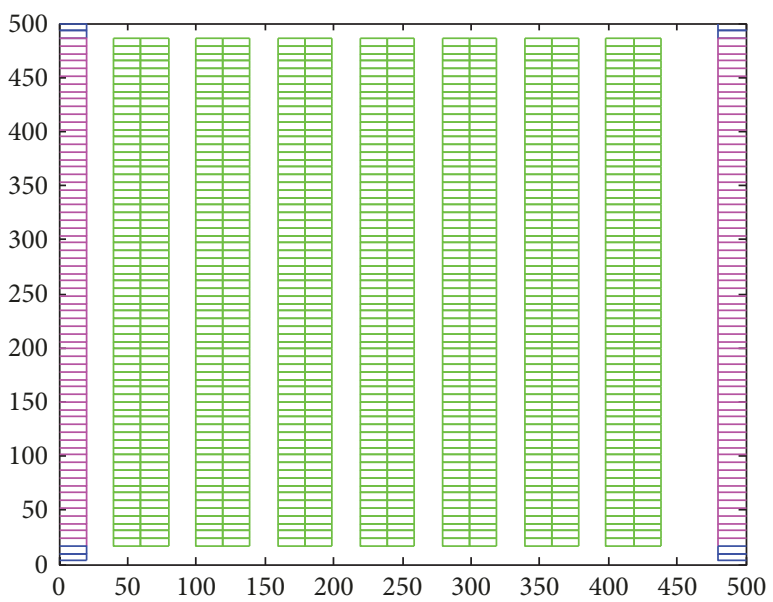

(e)

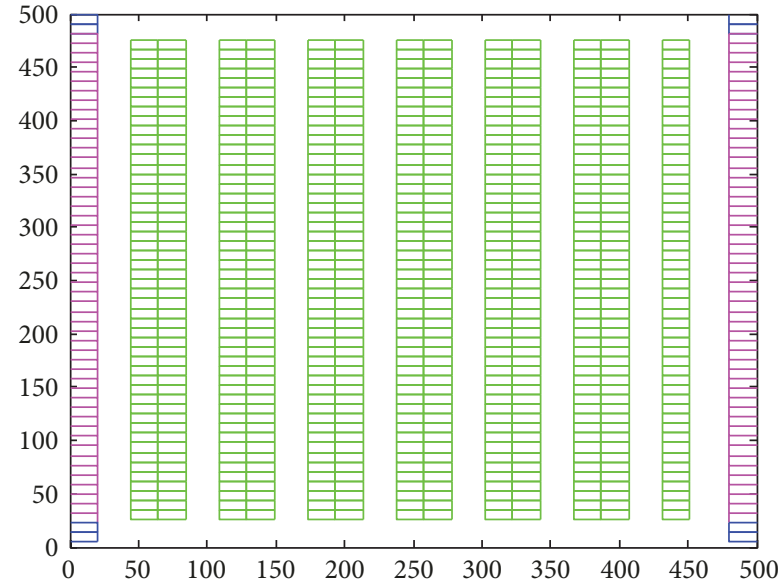

(b)

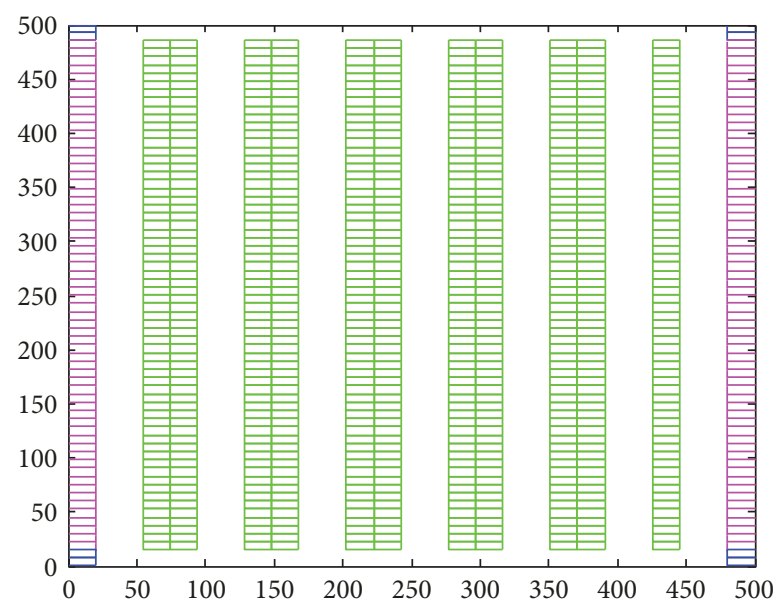

(c)

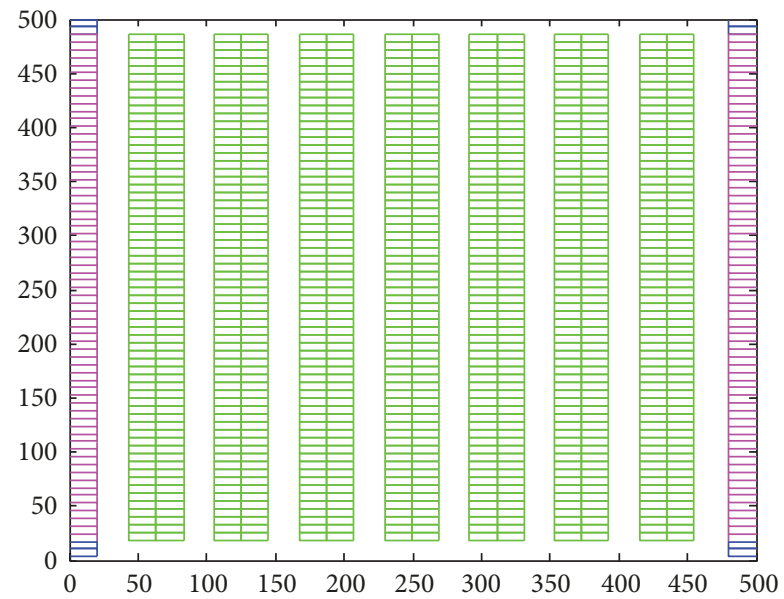

(d)

FIGURE 2: Optimal layouts for square facilities with 500' sides. Panel: (a) human "small” (790 parking spaces), (b) human "large" (760 spaces), (c) AV "front-in" (814 spaces), (d) AV "reverse-in" (1036 spaces), (e) AV “toing/froing" (1080 spaces), and (f) AV "translation" maneuvers (1080 spaces). 


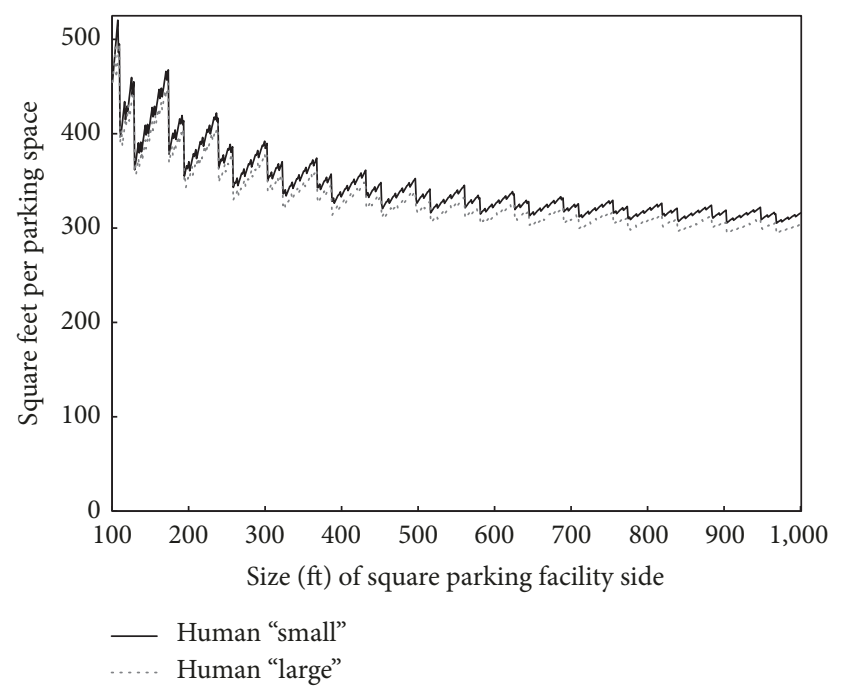

(a)

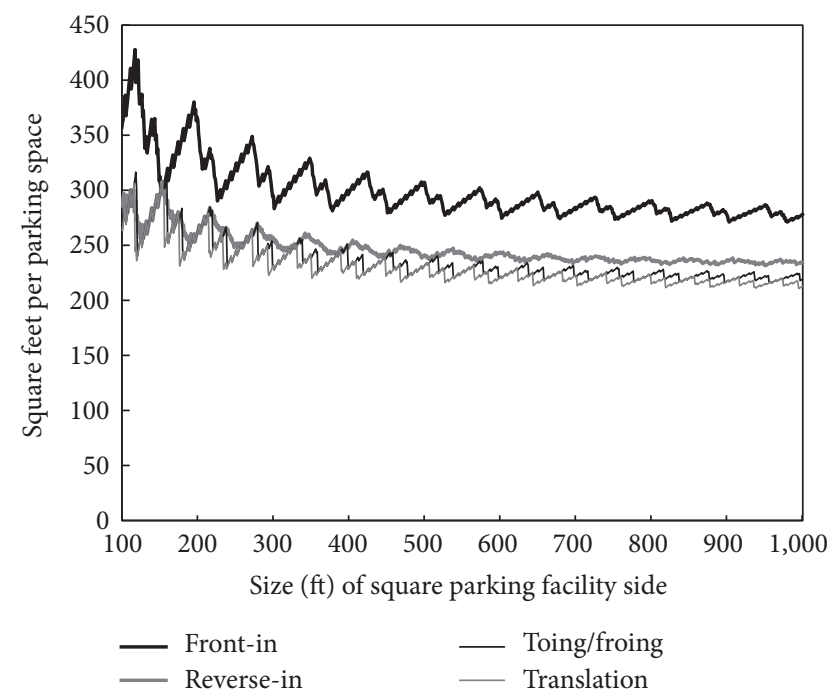

(b)

Figure 3: Area (sq. ft.) required per vehicle for square facilities with sides between $100^{\prime}$ and $1000^{\prime}$, in $1^{\prime}$ increments. (a) Human "small” and human "large"; (b) four AV maneuvers ("front-in," "reverse-in," "toing/froing," and "translation").

spaces designed for human drivers. (b) shows analogous curves for facilities designed for $\mathrm{AV}$ usage, using each of the four maneuvers. For both human and AV operations, it can be see that spatial efficiency increases with facility size (but subject to diminishing marginal returns) and that the sensitivity of efficiency to $1^{\prime}$-increment increases in facility size decreases with facility size. The latter effect is due to the addition of each marginal parking row or exterior parking space having a smaller proportional impact for larger facility sizes. Figure 3(b) also demonstrates near-monotonicity in the spatial efficiency of the AV maneuvers: "front-in" maneuvering is always much less efficient than "reverse-in" maneuvering. "Reverse-in" maneuvering is nearly always (for $98.8 \%$ of facility sizes) less efficient than "toing/froing"; the possibility of single-motion "reverse-in" maneuvering being more efficient than "toing/froing" is due to the reverse-in maneuver being able to use the area of the parking space to bypass the otherwise-strict requirement (with the exception of "translation") that the aisle width accommodates at a minimum the diagonal dimension of the vehicle. Finally, "translation" is always at least as efficient as "toing/froing"; in $59.6 \%$ of cases the two are equally efficient, and in the remaining $30.4 \%$ "translation" is more spatially efficient.

As noted in Section 3.2, we tested AV parking space widths ranging from $7^{\prime}$ to $21^{\prime}$, separately for each of the three types of spaces (type $\rho, \sigma, \tau$ ). In the numerical analysis, we found that, for "front-in" layouts, for the large majority of the 901 square facility sizes that we examined the maximum number of parking spaces derives from all types of parking spaces having widths of $7^{\prime}$. For the "front-in" parking layout, in $82.9 \%$ of the square facility sizes this combination ( $a_{\rho}=$ $7^{\prime}, a_{\sigma}=7^{\prime}, a_{\tau}=7^{\prime}$ ) yields the maximum number of parking spaces, larger than (or equal to) any other combination up to $a_{\rho}=21^{\prime}, a_{\sigma}=21^{\prime}, a_{\tau}=21^{\prime}$. For the "reverse-in" maneuver, however, this minimum-space width combination

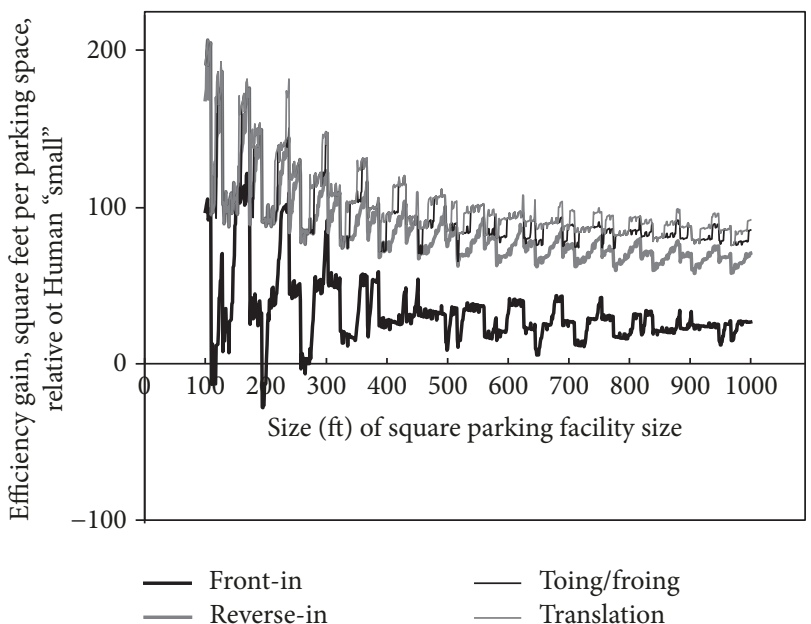

FIGURE 4: Difference in area (sq. ft.) required per vehicle, comparing human "small" parking space dimensions to the dimensions required for the four AV maneuvers ("front-in," "reverse-in," "toing/froing," and "translation"), for square facilities with sides between $100^{\prime}$ and $1000^{\prime}$, in $1^{\prime}$ increments.

$\left(a_{\rho}=7^{\prime}, a_{\sigma}=7^{\prime}, a_{\tau}=7^{\prime}\right)$ is only most efficient for a minority (24.8\%) of the 901 square sizes.

Figure 4 depicts the difference between each of the four $\mathrm{AV}$-operation scenarios and the minimum ("small") humandriving scenario. For large facility sizes all AV scenarios are more efficient spatially than human-driving; however for small facilities there are square facility sizes for which humandriving is more efficient than AV "front-in" operation. This arises from the minimum aisle width in the human-driving standards not permitting human drivers of the Passenger Car design vehicle to enter a parking space in a single motion. The curve for "toing/froing" in Figure 4 shows that when AVs are 


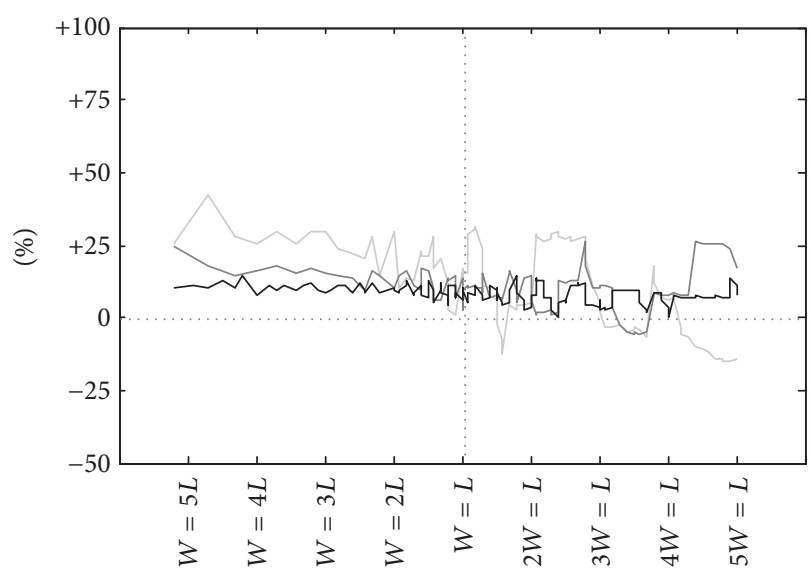

(a)

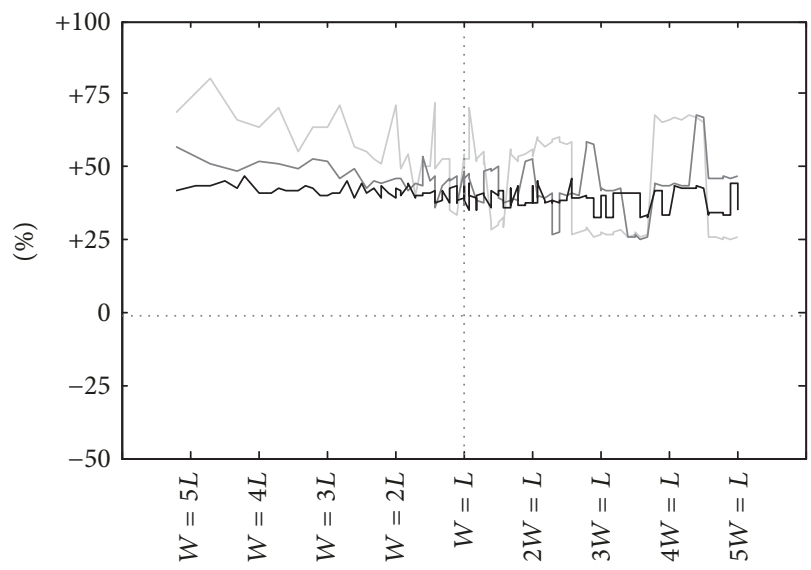

(d)

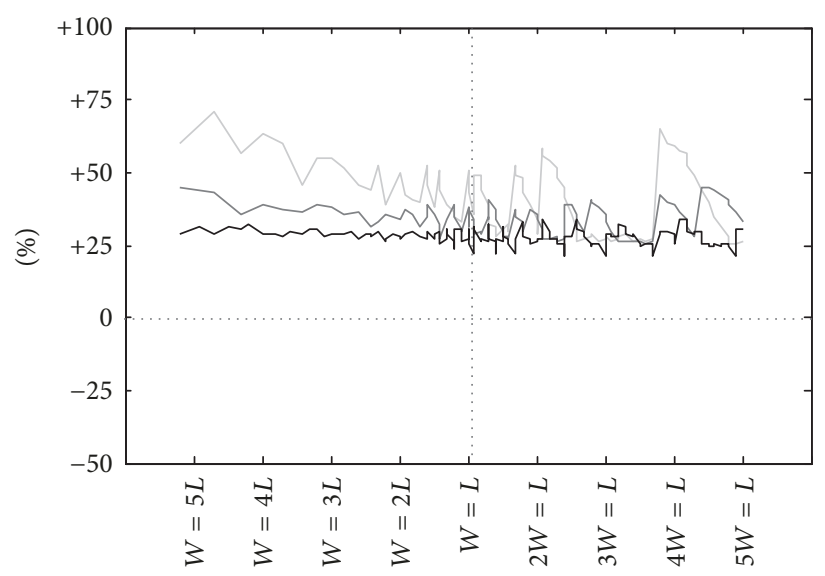

(b)

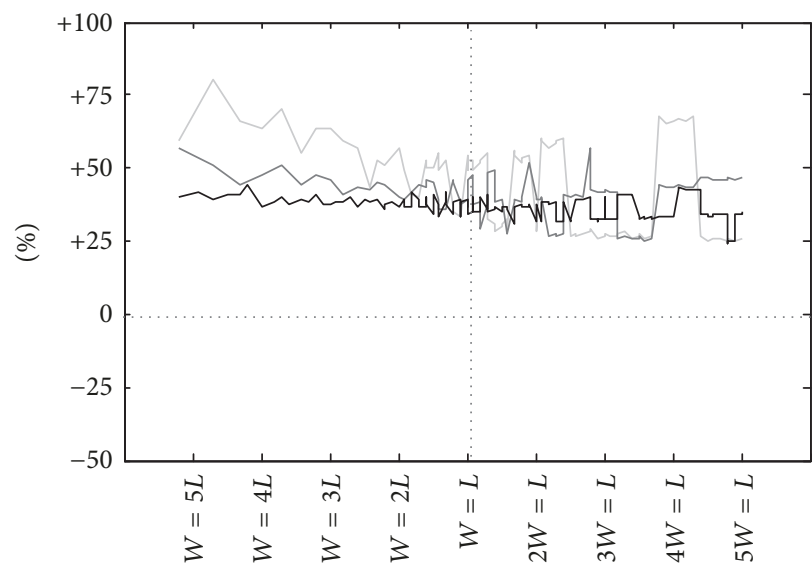

(c)

FIGURE 5: Percentage gain in efficiency (sq. ft. per parking space), relative to human "small" dimensions, for rectangular facilities with area of 62,500 (light gray curves), 250,000 (medium gray), and 1,000,000 (black) sq. ft. $x$-axis depicts ratio of facility length $(\dot{L})$ relative to width $(\dot{W})$. Panel: (a) AV "front-in," (b) AV “reverse-in," (c) AV “toing/froing," and (d) AV "translation" maneuvers.

TABLE 1: Percentage difference in parking capacity between two human parking space dimensions ("small" and "large") and dimensions required for the four AV maneuvers ("front-in," "reverse-in," "toing/froing," and "translation"). Values in each cell represent mean (standard deviation), calculated across square facilities with sides $100^{\prime}$ to $1000^{\prime}$ in $1^{\prime}$ increments.

\begin{tabular}{lcc}
\hline & Human "small" & Human "large" \\
\hline AV "front-in" & $+10.9 \%(6.9 \%)$ & $+15.0 \%(7.1 \%)$ \\
AV “reverse-in" & $+34.2 \%(7.9 \%)$ & $+39.1 \%(8.1 \%)$ \\
AV “toing/froing" & $+40.8 \%(8.3 \%)$ & $+45.9 \%(8.5 \%)$ \\
AV “translation" & $+44.0 \%(8.5 \%)$ & $+49.3 \%(8.7 \%)$ \\
\hline
\end{tabular}

also assumed to make multiple forward and reverse motions, spatial efficiency is improved relative to human drivers for all facility sizes. This quantifies what would be expected, due to our specification that AVs simply maneuver more precisely than human drivers, with all else held constant.

Table 1 shows the change in spatial efficiency (the proportional change in parking capacity) for square facilities averaged across squares with sides $100^{\prime}$ to $1000^{\prime}$ feet. It can be seen that efficiency gains range from approximately $7 \%$ for $\mathrm{AV}$ front-in maneuvers to $44 \%$ for the limiting case of the "translation" motion. These values arise from comparing AV maneuvers against "small" human parking space dimensions; the respective gains in efficiency relative to "large" human parking spaces are $15 \%$ and $49 \%$. A result of interest is that the large majority of the gains $(34 \% / 44 \% \approx 78 \%)$ can be achieved simply by designing parking facilities that assume that AVs will be instructed to perform single-motion "reverse-in" maneuvers to enter parking spaces. Of the residual efficiency gains after taking this into account, the majority $(41 \%-34 \% \approx$ $7 \%)$ are achievable by assuming forward-driving along circulation aisles as is done by humans and multiple (rather than a single) toing/froing motions. By contrast, the efficiency gains from pure "translation" motion, which require changes to automotive engineering practice, are relatively small $(44 \%-41 \% \approx 3 \%)$.

Tables 2 and 3 and Figure 5 show the effects of elongation in parking facility shape. Table 2 presents results analogous to those in Table 1 , for rectangular shapes of dimensions $\dot{W}=2 \dot{L}$ 
TABle 2: Percentage difference in parking capacity between two human parking space dimensions ("small" and "large") and dimensions required for the four AV maneuvers ("front-in," "reverse-in," "toing/froing," and "translation"). Values in each cell represent percentage differences for rectangles with $\dot{W}=2 \dot{L}$ having the same area as square facilities with sides of $250^{\prime} \times 250^{\prime}(62,500 \mathrm{sq} . \mathrm{ft}$. $)$, $500^{\prime} \times 500^{\prime}$ $(250,000$ sq. ft. $)$, and $1000^{\prime} \times 1000^{\prime}(1,000,000$ sq. ft. $)$.

\begin{tabular}{lcr}
\hline & Human "small” & Human "large" \\
\hline AV "front-in" & $+3.8 \%,+6.2 \%,+7.5 \%$ & $+6.7 \%,+10.5 \%,+11.7 \%$ \\
AV "reverse-in" & $+28.6 \%,+33.9 \%,+27.1 \%$ & $+32.2 \%,+39.3 \%,+32.1 \%$ \\
AV "toing/froing" & $+28.6 \%,+39.6 \%,+37.3 \%$ & $+32.2 \%,+45.3 \%,+42.7 \%$ \\
AV "translation" & $+53.5 \%,+52.2 \%,+43.2 \%$ & $+57.8 \%,+58.4 \%,+48.9 \%$ \\
\hline
\end{tabular}

TABle 3: Percentage difference in parking capacity between two human parking space dimensions ("small" and "large") and dimensions required for the four AV maneuvers ("front-in," "reverse-in," "toing/froing," and "translation"). Values in each cell represent percentage differences for rectangles with $\dot{L}=2 \dot{W}$ having the same area as square facilities with sides of $250^{\prime} \times 250^{\prime}\left(62,500 \mathrm{sq} . \mathrm{ft}\right.$.), $500^{\prime} \times 500^{\prime}$ $(250,000$ sq. ft. $)$, and $1000^{\prime} \times 1000^{\prime}(1,000,000$ sq. ft. $)$.

\begin{tabular}{lcr}
\hline & Human "small” & Human "large" \\
\hline AV "front-in" & $+29.6 \%,+10.0 \%,+9.7 \%$ & $+31.3 \%,+13.6 \%,+14.2 \%$ \\
AV “reverse-in" & $+50.0 \%,+34.2 \%,+27.5 \%$ & $+52.0 \%,+38.6 \%,+32.7 \%$ \\
AV "toing/froing" & $+57.2 \%,+40.3 \%,+36.4 \%$ & $+59.3 \%,+44.8 \%,+41.9 \%$ \\
AV "translation" & $+71.1 \%,+46.3 \%,+42.3 \%$ & $+73.3 \%,+51.1 \%,+48.1 \%$ \\
\hline
\end{tabular}

(i.e., the dimension longitudinally along the parking rows is one-half of the length of the perpendicular dimension); Table 3 is complementary to Table 2 but presents results for rectangles with the relationship $\dot{L}=2 \dot{W}$. Values in the cells of Table 2 refer to individual facility sizes with the same area as squares of sides $250^{\prime} \times 250^{\prime}(62,500 \mathrm{sq}$. ft. $), 500^{\prime} \times$ $500^{\prime}$ (250,000 sq. ft.), and $1000^{\prime} \times 1000^{\prime}(1,000,000$ sq. ft. $)$. In comparison to square facilities (Table 1), it can be seen, for instance, that the efficiency gains are smaller for all three rectangle sizes for AV "front-in" maneuvers, "reverse-in" maneuvers, and "toing/froing" maneuvers. For "translation" maneuvers, two smaller rectangles with this shape have larger gains in spatial efficiency than squares having the same area.

Unlike the rectangle-shapes analyzed in Table 2, for "front-in" and "reverse-in" maneuvers the spatial efficiency gains reported in Table 3 decrease monotonically from the 62,500 sq. $\mathrm{ft}$. facility to the $250,000 \mathrm{sq}$. ft. facility and then again to the $1,000,000 \mathrm{sq}$. $\mathrm{ft}$. facility. Figure 5 shows that this relationship is not strictly monotonic, though the efficiency gains tend to decrease with facility size. This is due to edge effects (which are different for different facility shapes) being increasingly dominated in larger facilities by interior parking activity away from the facility edges (which does not depend on facility shape).

\section{Conclusions}

In this study we develop and implement a mixed integer nonlinear optimization model to evaluate the impact of AVs' precise physical maneuvering on the spatial efficiency of surface parking facilities. We present numerical results considering three types of parking maneuvers ("front-in," "reverse-in," and "translation") and facility sizes from $100 \mathrm{ft}$ $\times 100 \mathrm{ft}(10,000 \mathrm{sq} f \mathrm{ft})$ to $1000 \mathrm{ft} \times 1000 \mathrm{ft}(1,000,000 \mathrm{sq} f \mathrm{ft})$, including both square and rectangular shapes of a surface parking facility. For both human and AV operations, it can be concluded that spatial efficiency increases with facility size (but subject to diminishing marginal returns) and that the sensitivity of efficiency to $1^{\prime}$-increment increases in facility size decreases with facility size. Among all the AV maneuvers, "front-in" maneuvering is always much less efficient than "reverse-in" maneuvering. "Reverse-in" maneuvering is nearly always (for $98.8 \%$ of facility sizes) less efficient than "toing/froing" maneuvering; the possibility of single-motion "reverse-in" maneuvering being more efficient than "toing/froing" maneuvering is due to the reverse-in maneuver being able to use the area of the parking space to bypass the otherwise-strict requirement (with the exception of "translation") that the aisle width accommodates at a minimum the diagonal dimension of the vehicle. Finally, "translation" maneuvering is always at least as efficient as "toing/froing" maneuvering; in 59.6\% of cases the two are equally efficient, and in the remaining 30.4\% "translation" maneuvering is more spatially efficient.

We demonstrate that, by comparing AV maneuvers against human parking space dimensions, the gains in efficiency relative to "large" human parking spaces are a minimum of $11 \%$ and a maximum of $49 \%$ and that the majority of the gains (approximately 78\%) are achievable simply by designing parking facilities that assume that AVs will be instructed to perform single-motion "reverse-in" maneuvers to enter parking spaces, even without major changes to automotive design (i.e., four pivoting wheels) or specifying that multiple toing-and-froing maneuvers must be performed when parking. Our objective in quantifying these first-order efficiency gains from AVs' parking is to advance towards the broader goal of establishing credible quantitative forecasts of how AVs will impact urban transport geography.

We now conclude this paper with a brief discussion of future research needed to advance this line of research. 
First, further research is needed to establish the efficiency impacts on parking facilities with irregular geometry or with internal obstructions. Fortunately, whereas Ricker [29] points out that the increasing size of automobiles in the mid20th century (and hence increasing spatial requirements for parking maneuvers) led to obsolescence of many parking garages with structural columns that prevented increasing the size of parking spaces, the opposite is the case from the stimulus of introducing AV technology: AVs will be able to maneuver into any parking spaces designed for human drivers to access, in addition to spaces with smaller dimensions.

Second, further research is needed to integrate the layout strategies presented in this study with vehicle-toinfrastructure and vehicle-to-vehicle communications of the type recently proposed by Estepa et al. [44]. The magnitude and distribution of the combined efficiency gains will be of interest. There is also a need to integrate analysis of pure spatial efficiency (as in the current study) with the efficiency of vehicular circulation. Layouts that are highly efficient spatially may have trade-offs with circulation efficiency; this trade-off requires quantification in the context of AVs.

Third, research is needed to extend from the single layout strategy that we studied (see Figure 1(a)) to consider other possibilities, including angled-parking, sites with internal obstructions (e.g., columns for multilevel parking facilities), and alternative plans for internal circulation. It would also be of interest to investigate options such as flexible (rather than defined by painted striping) definition of parking spaces and inclusion of different-sized spaces in the same facility, to accommodate heterogeneity in the size distribution of automobiles.

Finally, further enquiry is required to investigate the microeconomics of alternative AV operations for vehicle storage such as on-site vehicle parking, autonomous-driving to off-site parking facilities, and continuous on-street circulation in autonomous-driving mode.

Ultimately, for planning purposes there is a need for standardized quantitative methods that take the parking network of a city as an input and calculate both the aggregate (citywide) spatial efficiency impacts of AVs and the spatial distribution. This study is intended as an initial step towards this objective.

\section{Notations}

$$
\begin{array}{ll}
C_{\max }: \quad \begin{array}{l}
\text { Maximum capacity (integer number of } \\
\text { parking spaces) of a rectangular parking } \\
\text { facility conditional on the facility's length } \\
\text { and width }
\end{array} \\
\text { Width of the parking facility (east-west } \\
\text { dimension) } \\
\text { Length of the parking facility (north-south } \\
\text { dimension) } \\
\dot{L}: \quad \begin{array}{l}
\text { Number (integer) of parking spaces of types } \\
\rho, \sigma, \tau \text { generic form is } n_{i}
\end{array} \\
n_{\rho}, n_{\sigma}, \text { and } n_{\tau} \\
a_{\rho}, a_{\sigma}, \text { and } a_{\tau}: \begin{array}{l}
\text { Width of parking spaces of types } \rho, \sigma, \tau \\
\text { generic form is } a_{i}
\end{array}
\end{array}
$$

L: $\quad$ Length of parking spaces $\left(19.75^{\prime}\right)$

$w$ : Width of Passenger Car design vehicle $\left(7^{\prime}\right)$

$l$ : $\quad$ Length of Passenger Car design vehicle $\left(19^{\prime}\right)$

$X_{\rho}$ : Number (integer) of double-loaded rows of parking spaces: these rows contain type $\rho$ parking spaces

$X_{\rho, s}:$ Number (integer) of single-loaded interior rows of parking spaces: these rows contain type $\rho$ parking spaces

$X_{\epsilon, s}$ : Number (integer) of single-loaded exterior rows of parking spaces: the two parking spaces at each corner of the facility that align with the east-west circulation rows are type $\sigma$ parking spaces; all other parking spaces in these rows are type $\tau$.

$W_{9}$ : Width of north-south circulation aisles between exterior rows and interior rows

$W_{\mu}$ : Width of north-south circulation aisles between adjacent interior rows

$W_{\pi}$ : Width of east-west circulation aisles, along the northern and southern boundary

$Y_{\epsilon}:$ Minimum width of north-south circulation aisles to accommodate access to/from parking spaces of type $\tau$

$D_{f}$ : Minimum aisle width for front-in parking maneuvers

$D_{r}:$ Minimum aisle width for reverse-in parking maneuvers

$D_{t}$ : Minimum aisle width for translation parking maneuvers

$r$ : Minimum turning radius, inside rear wheel

$R$ : Minimum turning radius, outside point of front bumper

$R^{\prime}$ : Minimum turning radius, outside point of rear bumper

$\mathrm{O}_{s}$ : Side overhang (center of tire to side of vehicle)

$t$ : Tire "tread" (distance center to center of tires, along either rear or front axle).

\section{Disclosure}

Any errors in this paper are the authors' sole responsibility.

\section{Conflicts of Interest}

The authors declare that they have no conflicts of interest.

\section{Acknowledgments}

The authors acknowledge financial support from the National Natural Science Foundation of China (Project no. 71671147) and the University Transportation Research Center, Region 2 (Grant no. 49198-13-28: Simulation of Automated Vehicles' Drive Cycles). The authors wish to thank Peter Faith, P.E., and Michael Wieszchowski, P.E., P.T.O.E., of Greenman-Pedersen Inc. for helpful discussions. 


\section{References}

[1] D. J. Fagnant and K. M. Kockelman, "The travel and environmental implications of shared autonomous vehicles, using agent-based model scenarios," Transportation Research Part C: Emerging Technologies, vol. 40, pp. 1-13, 2014.

[2] V. Milanes, S. E. Shladover, J. Spring, C. Nowakowski, H. Kawazoe, and M. Nakamura, "Cooperative adaptive cruise control in real traffic situations," IEEE Transactions on Intelligent Transportation Systems, vol. 15, no. 1, pp. 296-305, 2014.

[3] A. Malokin, G. Circella, and P. Mokhtarian, "How do activities conducted while commuting influence mode choice? Testing public transportation advantage and autonomous vehicle scenarios," in Proceedings of the 94th Annual Meeting of the Transportation Research Board, Washington, DC, USA, 2015.

[4] S. Smith, J. Bellone, S. Bransfield et al., "Benefits estimation framework for automated vehicle operations," U.S. Dept. of Transportation ITS Joint Program Office Report \# FHWA-JPO16-229. Retrieved 1/1/18 via: https://ntl.bts.gov/lib/55000/55400/ 55443/AVBenefitFrameworkFinalReport082615_Cover1.pdf, 2015.

[5] A. Millard-Ball, "Pedestrians, Autonomous Vehicles, and Cities," Journal of Planning Education and Research, vol. 38, no. 1, pp. 6-12, 2016.

[6] NACTO, NACTO Policy Statement on Automated Vehicles, 2016, http://nacto.org/wp-content/uploads/2016/06/NACTO-PolicyAutomated-Vehicles-201606.pdf.

[7] S. Lam, J. Taghia, and J. Katupitiya, "Evaluation of a transportation system employing autonomous vehicles," Journal of Advanced Transportation, vol. 50, no. 8, pp. 2266-2287, 2016.

[8] Z. Wadud, D. MacKenzie, and P. Leiby, "Help or hindrance? The travel, energy and carbon impacts of highly automated vehicles," Transportation Research Part A: Policy and Practice, vol. 86, pp. 1-18, 2016.

[9] M. Gucwa, "Mobility and energy impacts of automated cars," in Proceedings of the Presentation at Automated Vehicles Symposium, San Francisco, Calif, USA, July 2014, http://higherlogicdownload.s3.amazonaws.com/AUVSI/3a47c2f1-97a8-4fb78a39-56cba0733145/UploadedImages/documents/pdfs/7-16-14\% 20AVS\%20presentations/Michael\%20Gucwa.pdf.

[10] J. R. Healey and K. Mays, "Which cars park themselves best? Challenge results," USA Today, Article ID 1743199, 2012, https:// www.usatoday.com/story/money/cars/2012/12/06/self-parkingcars-challenge//via.

[11] R. Vincent, "When car ownership fades, this parking garage will be ready for its next life," Los Angeles Times, Article ID 20170405, 2017, http://www.latimes.com/business/la-fi-carfuture-real-estate-20170405-story.html.

[12] M. Manville and D. Shoup, "Parking, people, and cities," Journal of Urban Planning and Development, vol. 131, no. 4, pp. 233-245, 2005.

[13] C. McCahill, J. Haerter-Ratchford, N. Garrick, and C. AtkinsonPalombo, "Parking in Urban Centers: Policies, supplies, and implications in six cities," Transportation Research Record, vol. 2469, pp. 49-56, 2014.

[14] National Parking Association, Parking in America, Washington, DC, USA, 2008.

[15] D. J. Sun, X.-Y. Ni, and L.-H. Zhang, "A discriminated release strategy for parking variable message sign display problem using agent-based simulation," IEEE Transactions on Intelligent Transportation Systems, vol. 17, no. 1, pp. 38-47, 2016.
[16] X. Y. Ni and D. J. Sun, "Agent-based modelling and simulation to assess the impact of parking reservation system," Journal of Advanced Transportation, 2017.

[17] A. Horni, L. Montini, R. A. Waraich, and K. W. Axhausen, "An agent-based cellular automaton cruising-forparking simulation," Transportation Letters, vol. 5, no. 4, pp. 167-174, 2013.

[18] H. Dia, "An agent-based approach to modelling driver route choice behaviour under the influence of real-time information," Transportation Research Part C: Emerging Technologies, vol. 10, no. 5-6, pp. 331-349, 2002.

[19] Y. Geng and C. G. Cassandras, "New 'smart parking' system based on resource allocation and reservations," IEEE Transactions on Intelligent Transportation Systems, vol. 14, no. 3, pp. 1129-1139, 2013.

[20] M. Dayan, "System and method for determining and reserving available parking," U.S. Patent Application 2011/0022427, 2011.

[21] U. Levine, A. Shinar, and E. Shabtai, "System and method for parking time estimations," US Patent7, 936, 284, 2011.

[22] F. F. Kuhlman, D. H. R. Sarma, and A. P. Harback, "Vehicle parking spot locator system and method using connected vehicles," U.S. Patent Application 2012/0056758, 2012.

[23] H. Belzner and P. Pedron, "Method for processing measurement data of a vehicle in order to determine the start of a search for a parking space," U.S. Patent Application 2016/0210860, 2014.

[24] S. R. Urbach, "Systems and methods for determining parking difficulty of segments of a geographic area," US Patent 8,847 , 791,2014

[25] L. D. Elie and D. S. Rhode, "System and method for autonomous valet parking using plenoptic cameras," U.S. Patent US9557741B1, 2017.

[26] Nissan Motor Company Ltd. (n.d.) Concept Car Pivo 2. Retrieved 1/1/18 via: http://www.nissanglobal.com/EN/PIVO2/ index.html.

[27] M. Kroger, Auto-Studien: Wenn Visionen Wirklichkeit warden (Automotive studies: when vision becomes reality), http:// www.spiegel.de/fotostrecke/alte-konzeptautos-frueher-sciencefiction-heute-realitaet-fotostrecke-86469-2.html, 2012.

[28] A. Schaub, J. C. R. de la Cruz, and D. Burschka, "Autonomous parking using a highly maneuverable robotic vehicle," in Proceedings of the 19th International Federation of Automatic Control (IFAC) World Congress, Cape Town, South Africa, 2014, http://www6.in.tum.de/Main/Publications/Schaub14.pdf.

[29] E. R. Ricker, Traffic Design of Parking Garages, Eno Foundation. Columbia University Press, New York, NY, USA, 1948.

[30] C. S. Chen and P. Schonfeld, "Optimum stall angle for large parking lots," Journal of Transportation Engineering, vol. 114, no. 5, pp. 574-583, 1988.

[31] R. Iranpour and D. Tung, "Methodology for optimal design of a parking lot," Journal of Transportation Engineering, vol. 115, no. 2, pp. 139-160, 1989.

[32] I. L. Chodash, "Relative efficiencies of various parking angles," ITE Journal, 1986.

[33] S. Blackburn, “The geometry of perfect parking. Working Paper commissioned by Vauxhall Motors," Retrieved, vol. 1/1/18, 2009, http://personal.rhul.ac.uk/uhah/058/perfect_parking.pdfvia.

[34] E. Ferguson, "Zoning for parking as policy process: A historical review," Transport Reviews, vol. 24, no. 2, pp. 177-194, 2004.

[35] D. Shoup, The High Cost of Free Parking, Planners Press, Chicago, Ill, USA, 2nd edition, 2011. 
[36] X.-Y. Ni, D. Sun, and Z.-R. Peng, "An improved incremental assignment model for parking variable message sign location problem," Journal of Advanced Transportation, vol. 49, no. 7, pp. 817-828, 2015.

[37] R. Arnott, E. Inci, and J. Rowse, "Downtown curbside parking capacity," Journal of Urban Economics, vol. 86, pp. 83-97, 2015.

[38] M. Chester, A. Fraser, J. Matute, C. Flower, and R. Pendyala, "Parking Infrastructure: A Constraint on or Opportunity for Urban Redevelopment? A Study of Los Angeles County Parking Supply and Growth," Journal of the American Planning Association, vol. 81, no. 4, pp. 268-286, 2015.

[39] Q. Chen, A. Conway, and J. Cheng, "Parking for residential delivery in New York City: Regulations and behavior," Transport Policy, vol. 54, pp. 53-60, 2017.

[40] K. Kim, G. Rousseau, J. Freedman, and J. Nicholson, "The Travel Impact of Autonomous Vehicles in Metro Atlanta through Activity-Based Modeling," in Proceedings of the 15th Annual Transportation Applications Conference, 2015, http:// www.trbappcon.org/2015conf/presentations/197_Autonomous\% 20Vehicles_Kyeil\%20Kim_Final.pptx.via.

[41] S. Childress, B. Nichols, B. Charlton, and S. Coe, "Using an activity-based model to explore possible impacts of automated vehicles," in Proceedings of the 94th Annual Meeting of the Transportation Research Board, Washington, DC, USA, 2015.

[42] M. Ferreira, L. Damas, H. Conceicao et al., "Self-automated parking lots for autonomous vehicles based on vehicular ad hoc networking," in Proceedings of the 25th IEEE Intelligent Vehicles Symposium, IV 2014, pp. 472-479, USA, June 2014.

[43] J. Timpner, S. Friedrichs, J. Van Balen, and L. Wolf, "KStacks: high-density valet parking for automated vehicles," in Proceedings of the IEEE Intelligent Vehicles Symposium, IV 2015, pp. 895-900, Seoul, South Korea, July 2015.

[44] R. Estepa, A. Estepa, J. Wideberg, M. Jonasson, and A. StenssonTrigell, "More Effective Use of Urban Space by Autonomous Double Parking," Journal of Advanced Transportation, vol. 2017, pp. 1-10, 2017.

[45] American Association of State Highway and Transportation Officials (AASHTO), A Policy on Geometric Design of Highways and Streets, 6th edition, 2011.

[46] Urban Land Institute (ULI), The Dimensions of Parking, National Parking Association, 5th edition, 2010.

[47] LINDO Software for Integer Programming, Retrieved 1/1/18 via: http://www.lindo.com/index.php/.

[48] M. Padberg and G. Rinaldi, "A branch-and-cut algorithm for the resolution of large-scale symmetric traveling salesman problems," SIAM Review, vol. 33, no. 1, pp. 60-100, 1991.

[49] M. R. Bussieck and S. Vigerske, MINLP Solver Software, Wiley Encyclopedia of Operations Research and Management Science, 2010. 


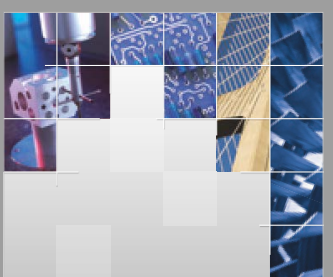

\section{Enfincering}
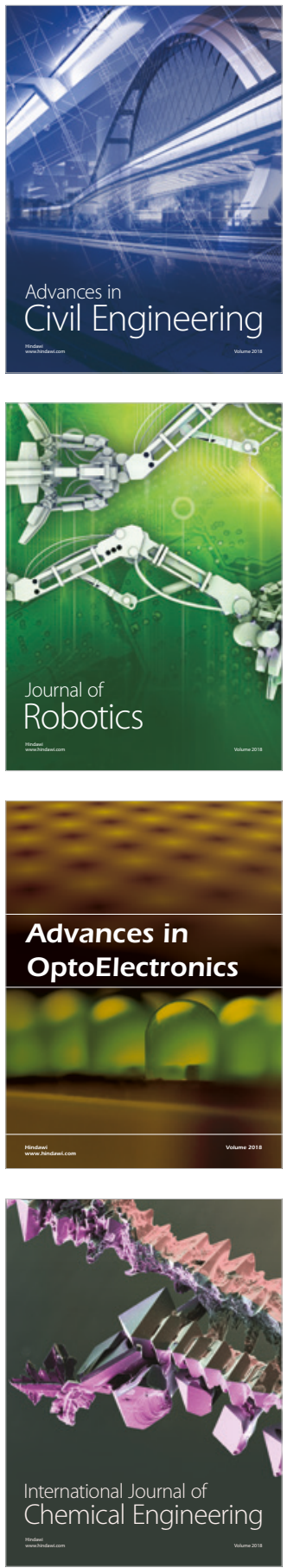

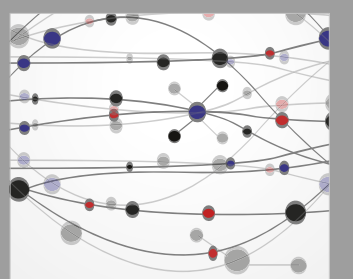

\section{Rotating \\ Machinery}

The Scientific World Journal

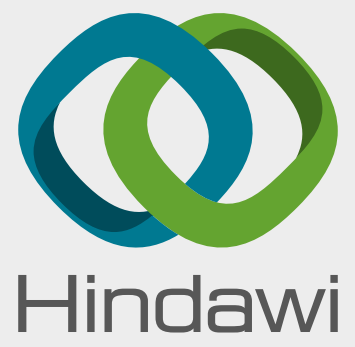

Submit your manuscripts at

www.hindawi.com
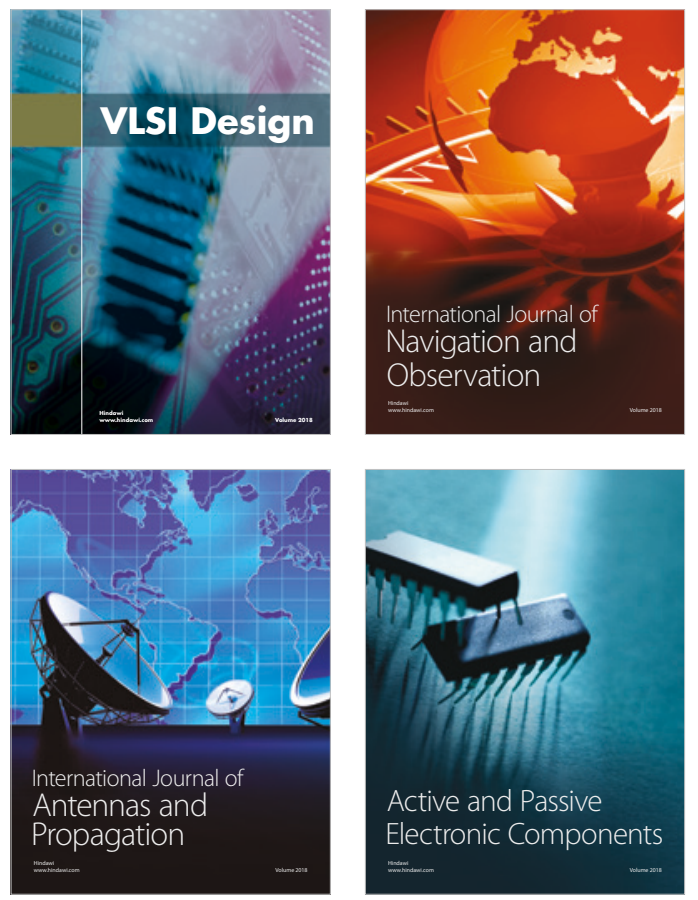
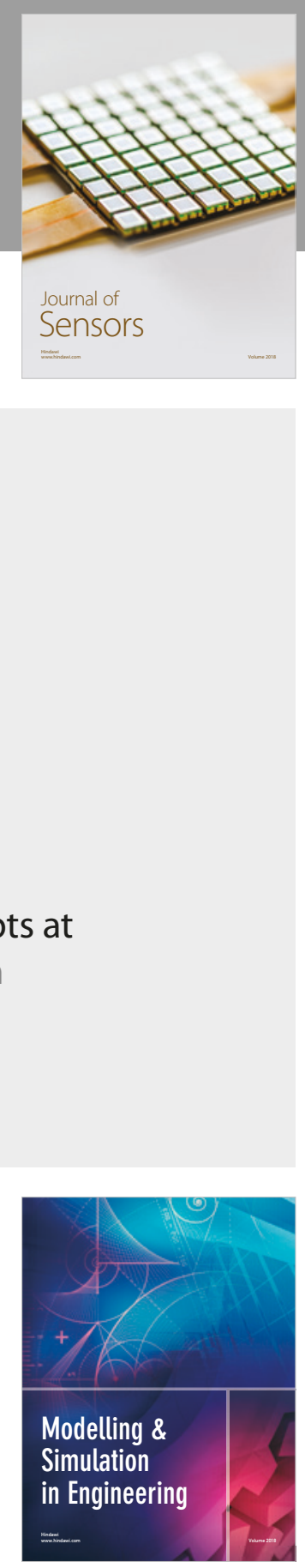

\section{Advances \\ Multimedia}
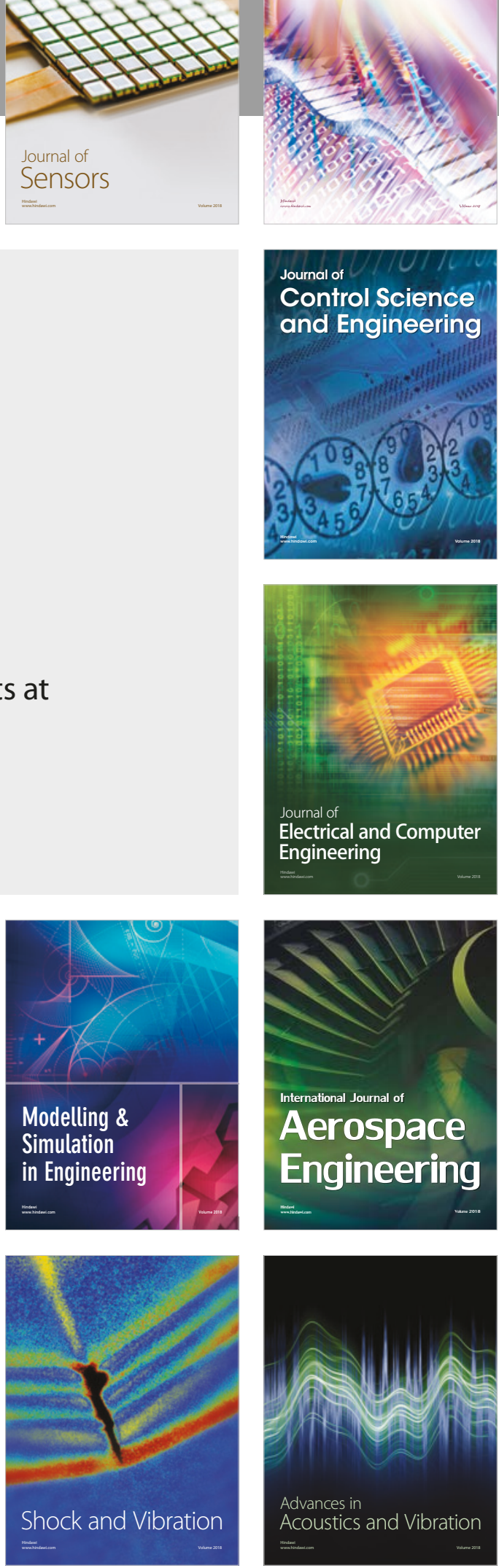